\title{
LE CYCLE BIOCHIMIQUE DANS LES EAUX DOUCES
}

\author{
Par J. A. TIMMERMANS \\ Ingénieur des Eaux et Forêts \\ Station de Recherches des Eaux et Forets (Groenendaal-Belgique)
}

La compréhension des phénomènes biochimiques qui se déroulent dans l'eau, de même qu'une interprétation claire de la productivité piscicole d'une eau naturelle, ne sont possibles que si l'on connait les différents éléments, phases et transformations qui interviennent dans le cycle de cette vie aquatique et mènent au stade qui nous intéresse avant tout : le poisson.

Ce cycle biochimique complexe comprend plusieurs phases. Les éléments indispensables à l'amorçage du cycle, sont les matières nutritives en solution dans l'eau.

Nous donnons de ce cycle un schéma suggestif et aussi complet que possible, ce qui permettra de décrire plus clairement un phénomène qui est à la base de toute exploitation piscicole où intervient la nourriture naturelle.

\section{A. - Les phases du cycle biochimique.}

\section{Interprétation scientifique.}

Matières nutritives et énergie. - Les matières nutritives en solution dans l'eau proviennent :

10 Des terrains avec lesquels les eaux sont en contact.

20 Des précipitations atmosphériques.

3o De la minéralisation par les bactéries de détritus accumulés dans l'eau et produits dans celle-ci ou apportés de l'extérieur,

$4^{\circ}$ De sources extérieures : industrie, agriculture, agglomérations.

Grâce à l'énergie dispensée par le soleil sous forme de lumière et de chaleur, ces substances minérales, y compris l'acide carbonique présent dans l'eau, sont transformées par la végétation verte en matières organiques. Certaines plantes, les saprophytes, se nourrissent de détritus organiques.

Flore aquatique. - La végétation aquatique verte comprend les plantes inférieures en suspension dans l'eau (phytoplancton) ou recou- 
vrant les differents supports du milieu aquatique (couvertures biologiques) et les plantes supérieures : submergées, flottantes et palustres.

Faune herbivore. - Au cours de la phase suivante, une partie de cette végétation est consommée, à l'état vivant ou à l'état mort, par la faune herbivore (faune aquatique inférieure et poissons). Une autre partie très importante est réintroduite dans le cycle biochimique sous forme de détritus, minéralisés par les bactéries; elle restitue ainsi des éléments nutritifs et de l'énergie.

Faune carnivore. - Durant cette phase, une fraction de la faune herbivore est consommée par la faune carnivore, laquelle comprend aussi bien des organismes inférieurs que les poissons voraces. Comme pour la végétation, la faune herbivore qui échappe à la faune vorace est réintroduite dans le cycle après sa mort grâce à l'activité bactérienne.

A l'intérieur de la faune carnivore, les organismes plus faibles ou plus petits forment, à l'état vivant ou à l'état mort, une partie de la nourriture des autres voraces. Ici, de nouveau, le cycle biochimique récupère, grâce à la minéralisation, des matières nutritives et de l'énergie provenant de la matière animale morte.

La production piscicole, que ce soit au niveau de la faune herbivore ou à celui de la faune carnivore, est fonction de la multiplication et du développement des organismes vivants au cours des phases précédentes et est donc nécessairement limitée par l'importance du peuplement biologique de l'eau.

Minéralisation. - Une phase métabolique essentielle du cycle biochimique dans l'eau est la minéralisation effectuée par les bactéries. Les détritus accumulés par la mort de la flore, de la faune et des poissons, ainsi que les détritus venant de l'extérieur sont décomposés, grâce aux bactéries et réintroduits dans le cycle biochimique sous forme de composants plus simples, utilisables dans l'assimilation des plantes vertes. Par la décomposition bactérienne, la vase du fond et les détritus constituent un vrai laboratoire qui, d'ailleurs, joue un rôle prépondérant dans l'auto-épuration d'une eau. C'est là que s'accomplissent les phénomènes de restitution minérale qui entretiennent la richesse de l'eau en éléments biogènes.

Il faut ajouter à cet apport d'éléments, ceux provenant des excréments de l'ensemble de la faune aquatique, y compris les poissons.

\section{Interprétation économique.}

La distinction entre poissons herbivores et carnivores appartenant respectivement à deux phases distinctes et successives du biocycle, appartient plus spécialement au domaine scientifique et retient moins l'attention de celui qui s'intéresse à la productivité économique de l'eau, c'est-à-dire à la masse totale de poissons que peut donner une eau sur une surface et dans un temps déterminés.

Dans une interprétation économique et pour des raisons commodes, on peut également concevoir que l'ensemble du peuplement piscicole 
constitue, en fait, la phase la plus noble du cycle biologique, en quelque sorte celle de la production piscicole qui intéresse immédiatement l'utilisateur.

Nous dirons encore qu'en nous plaçant à ce même point de vue économique, la faune aquatique nutritive constitue la phase précédente et englobe les organismes nageant ou en suspension dans l'eau (zooplancton) et ceux vivant sur ou dans le fond, parmi la végétation et les détritus.

A l'intérieur de la population piscicole on distingue grosso modo les poissons-proies (blanchaille) et les poissons voraces, entre lesquels existe un certain équilibre dans les conditions naturelles.

C'est d'après cette conception économique que nous avons composé le schéma du cycle biochimique de l'eau douce.

Dans une eau non exploitée par l'homme, la mortalité naturelle fait rentrer l'élément poisson dans le cycle biochimique sous forme de détritus, tandis que les prédateurs enlèvent une partie vivante de cette production.

L'exploitation d'une eau parl'homme, soit par la péche professionnelle ou sportive, soit par les deux, enlève une partie de la production piscicole. Cette exploitation serait idéale si la totalité de la production était enlevée régulièrement sous forme de poissons intéressants.

\section{3. - Evolution quantitative de la matière produite.}

Il a été dit précédemment que la production piscicole est fonction du développement de la faune et de la flore aquatiques.

Chaque phase du cycle biochimique est ainsi directement en relation avec la phase précédente. Toutefois, au cours de la progression du cycle, il s'opère une diminution de la quantité de la matière vivante produite dans chaque phase. Ainsi la masse et le nombre d'individus de la faune herbivore sont moins importants que la masse et le nombre d'individus de la flore aquatique. Le même phénomène se réalise pour la succession de la faune carnivore à la faune herbivore. C'est d'ailleurs le cas bien connu à l'intérieur d'une population piscicole où le nombre et la masse des poissons-proies sont beaucoup plus importants que ceux des poissons voraces.

Cette dégradation quantitative de la matière produite est figurée dans le schéma par l'épaisseur des flèches qui relient entre elles les différentes phases du cycle biochimique.

\section{B. - L'influence de L'homme SUR LE GyCle BIOchimique.}

Selon la nature des terrains qu'elles traversent, les eaux piscicoles sont plus ou moins riches en matières nutritives minérales, ce qui conditionne dès le départ une productivité plus ou moins grande en poissons.

Une amélioration rationnelle de la qualité de l'eau, comme c'est souvent le cas en pisciculture par l'utilisation d'engrais et de fumier, n'est 


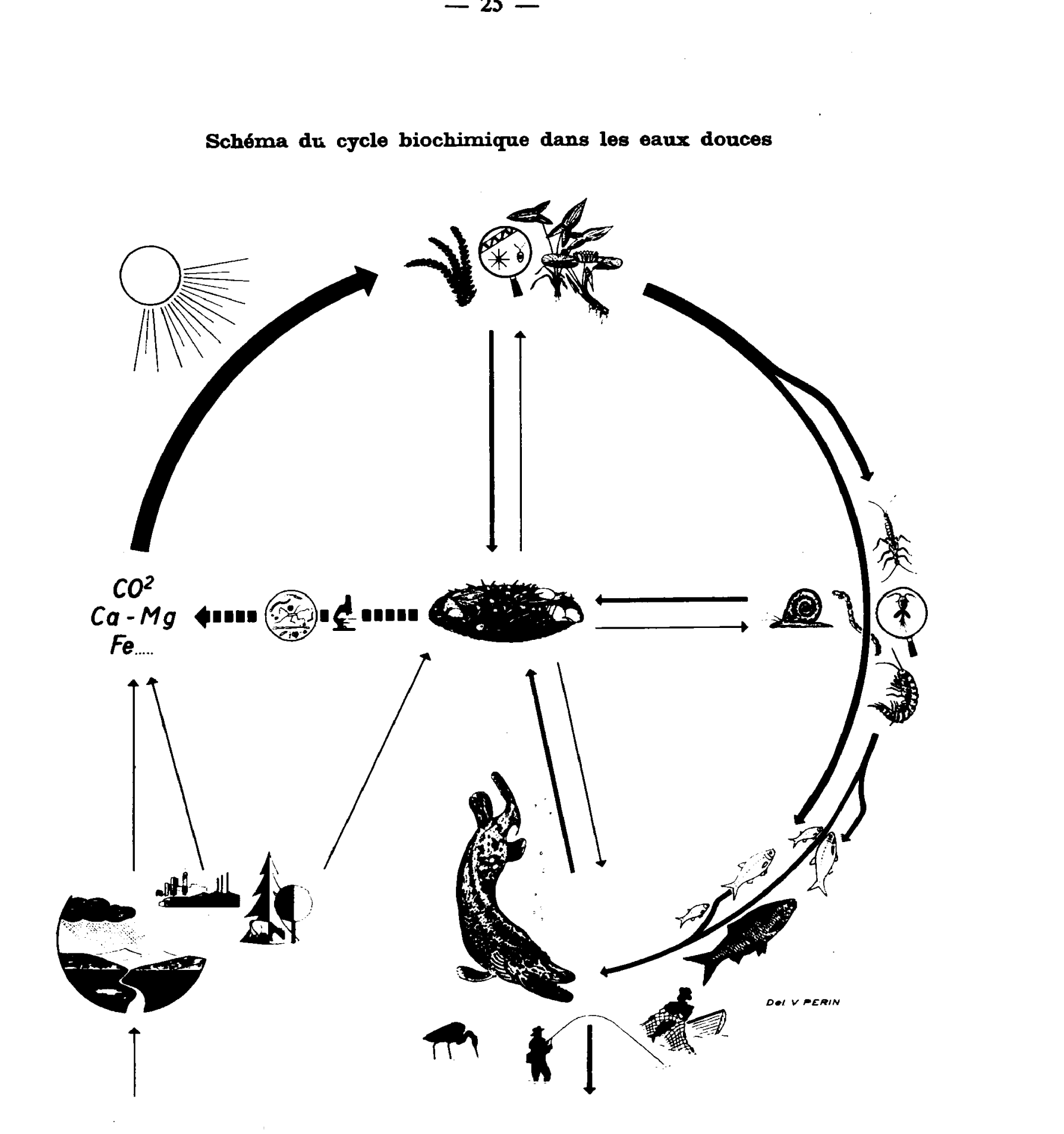


pas réalisable dans les eaux courantes et n'est faite que très exceptionnellement pour les eaux stagnantes naturelles.

Si une légère pollution rurale peut améliorer la qualité d'une eau pauvre, la plupart des déversements d'eaux usées nuisent à la production finale soit directement par des matières toxiques, soit indirectement par une surabondance de matières organiques.

Dans les eaux naturelles, la végétation aquatique est en général difficilement influençable par l'homme. Elle peut l'être indirectement par des eaux usées comme c'est le cas pour certaines algues qui prolifèrent grâce au déversement d'eau d'égouts. Quelquefois, un contrôle partiel de la végétation aquatique se fait, soit pour faciliter l'exercice de la pêche, soit pour empêcher un envahissement considérable devenant nuisible aux poissons. Le long de cours d'eau canalisés, l'introduction d'une bande de végétation palustre pour la protection des berges en terre est également profitable à la faune piscicole. Certaines plantes aquatiques, comme les roseaux, peuvent être exploitées directement par l'homme.

La chaleur et la lumière influencent grandement le développement de la végétation.

Dans les pays chauds la production piscicole, par l'intermédiaire de la végétation et de la faune aquatiques, est généralement plus élevée par unité de surface que dans les pays tempérés et ceci grâce à une température plus élevée. En ce domaine, l'homme peut influencer la température de l'eau par des déversements d'eaux usées.

Le facteur lumière peut subir des influences défavorables comme c'est le cas, par exemple, par la plantation d'épicéas et d'autres résineux en bordure immédiate de petits cours d'eau. Il peut être influencé favorablement par des nettoiements judicieux dans la végétation rivulaire.

La faune nutritive aquatique peut être influencée indirectement grâce à des améliorations du lit de certains cours d'eau, par la création de gouffres, par le relèvement du plan d'eau, par l'installation de déflecteurs et en général par la lutte contre l'ensablement.

Les cours d'eau canalisés ou rectifiés pour les besoins de la navigation ou de l'agriculture sont peu adaptés à la vie piscicole. Des améliorations peuvent certainement être étudiées dans chaque cas, sans qu'il $\mathrm{y}$ ait préjudice aux utilisations principales.

En supposant que les premières phases du cycle biochimique fonctionnent parfaitement, l'homme profite au maximum de la production finale s'il enlève régulièrement cette production sous forme de poissons intéressants, sans toucher au capital " poisson ". Il y tend par une réglementation adéquate, par la préservation des possibilités de reproduction pour les poissons et par un contrôle des prédateurs.

Dans le cas d'un déséquilibre entre les différentes espèces, de pèches trop intensives, ou d'absence de poissons intéressants, l'homme intervient par des déversements ou par l'introduction de nouvelles espèces. 


\section{Conclusions.}

Dans le cas de l'exploitation d'une eau piscicole naturelle, il ne suffit pas de porter uniquement son attention sur le produit final : le poisson. S'il faut voir une population piscicole comme une communauté avec son équilibre intérieur, il faut voir aussi une eau dans son ensemble et connaître les différents facteurs qui mènent finalement à cette production qui nous intéresse avant tout. Très souvent, l'homme intervient de façon néfaste au cours des différentes phases du cycle biochimique. Certaines influences comme la pollution posent un probleme ardu et complexe. D'autres influences, comme la pêche et les déversements sont plus faciles à contrôler, tandis que, pour de nombreuses eaux, l'influence humaine peut apporter des améliorations non négligeables. 